\title{
The influence of APOE and TOMM40 polymorphisms on hippocampal volume and episodic memory in old age
}

\section{Beata Ferencz ${ }^{1}$, Erika J. Laukka ${ }^{1}$, Martin Lövdén ${ }^{1,2}$, Grégoria Kalpouzos ${ }^{1}$, Lina Keller ${ }^{1,3}$, Caroline Graff ${ }^{3,4}$, Lars-Olof Wahlund ${ }^{5}$, Laura Fratiglioni ${ }^{1}$ and Lars Bäckman ${ }^{1}$}

${ }^{1}$ Aging Research Center, Karolinska Institutet and Stockholm University, Stockholm, Sweden

2 Department of Psychology, Lund University, Lund, Sweden

${ }^{3}$ KI-Alzheimer Disease Research Center, Karolinska Institutet, Stockholm, Sweden

${ }^{4}$ Department of Geriatrics, Karolinska University Hospital, Stockholm, Sweden

${ }^{5}$ Division of Clinical Geriatrics, Karolinska Institutet, Stockholm, Sweden

Edited by:

John J. Foxe, Albert Einstein College of Medicine, USA

Reviewed by:

Elena Galea, Universitat Autònoma de Barcelona, Spain

Natalie Nelissen, Katholieke

Universiteit Leuven, Belgium

Lei Niu, Albert Einstein College of

Medicine, USA

\section{*Correspondence:}

Beata Ferencz, Aging Research Center, Karolinska Institutet and Stockholm University, Gävlegatan 16, 11330 Stockholm, Sweden. e-mail: beata.ferencz@ki.se
Mitochondrial dysfunction is implicated in neurodegenerative disorders, such as Alzheimer's disease (AD). Translocase of outer mitochondrial membrane 40 (TOMM40) may be influential in this regard by influencing mitochondrial neurotoxicity. Little is known about the influence of the TOMM40 gene on hippocampal $(\mathrm{HC})$ volume and episodic memory (EM), particularly in healthy older adults. Thus, we sought to discern the influence of TOMM40 single nucleotide polymorphisms (SNPs), which have previously been associated with medial temporal lobe integrity (rs11556505 and rs2075650), on HC volume and EM. The study sample consisted of individuals from the Swedish National Study on Aging and Care in Kungsholmen (SNAC-K) who were free of dementia and known neurological disorders, and $60-87$ years of age $(n=424)$. EM was measured by using a 16-item word list with a 2-min free recall period and delineation of the HC was performed manually. The influence of Apolipoprotein E (APOE) and TOMM4O was assessed by $2 \times 2$ ANOVAs and partial correlations. There was no effect of APOE and TOMM40 on EM performance and $\mathrm{HC}$ volume. However, partial correlations revealed that $\mathrm{HC}$ volume was positively associated with free recall performance $(r=0.21, p<0.01$, $\left.r^{2}=0.04\right)$. When further stratified for TOMM40, the observed association between HC volume and free recall in APOE \&4 carriers was present in combination with TOMM40 rs 11556505 any $T\left(r=0.28, p<0.01, R^{2}=0.08\right)$ and $r s 2075650$ any $\mathrm{G}(r=0.28, p<0.01$, $\left.R^{2}=0.08\right)$ "risk" alleles. This pattern might reflect higher reliance on $\mathrm{HC}$ volume for adequate EM performance among APOE \&4 carriers with additional TOMM 40 "risk" alleles suggesting that the TOMM4O gene cannot merely be considered a marker of $A P O E$ genotype. Nevertheless, neither APOE nor TOMM40 influenced HC volume or EM in this population-based sample of cognitively intact individuals over the age of 60 .

Keywords: APOE, TOMM40, episodic memory, hippocampus, cognitive aging

\section{INTRODUCTION}

Although much is known about the clinical manifestations of Alzheimer's disease (AD), the etiology and pathophysiology of this disease still remains to be determined. Mitochondrial dysfunction is becoming increasingly highlighted in both pathological and non-pathological aging (Lin and Beal, 2006; Reddy et al., 2010; Swerdlow, 2011; Ferencz et al., 2012). Previously only considered in unison with $A P O E$, due to linkage disequilibrium (LD) between the two genes, TOMM40 was recently found to independently influence age of onset of AD (Roses et al., 2010; Li et al., 2012). In addition TOMM40 has been associated with risk of AD (Grupe et al., 2007; Abraham et al., 2008; Harold et al., 2009). However, these findings have not been consistently replicated (Cruchaga et al., 2011; Guerreiro and Hardy, 2012; Jun et al., 2012) and it is unclear if the effect can be attributed to TOMM40 or $A P O E$ as the two genes are in strong LD. Moreover, there is limited information on the influence of other TOMM40 SNPs, besides the rs10524523 poly- $\mathrm{T}$ length, on age- and $\mathrm{AD}$-sensitive markers, such as hippocampal (HC) volume and episodic memory (EM). Thus, further research is required in normal old adults before we can tease out the influence of genetic variation in $A P O E$ and TOMM40 on HC volume and EM memory, irrespective of dementia.

TOMM40, codes for the outer mitochondrial membrane pore subunit (TOM40), which is a pore through which proteins readily enter the mitochondria (Humphries et al., 2005). Known as the "powerhouse" of all cells, mitochondria serve an important function in the central nervous system, providing energy essential for neuronal survival (McBride et al., 2006; Baloyannis, 2011). Not always beneficial to the neurons, an increased net production of reactive oxygen species in mitochondria has been implicated in both pathological and non-pathological aging (Lin and Beal, 2006). Moreover, morphological changes within the mitochondria of the hippocampus have been observed in postmortem $\mathrm{AD}$ (Hirai et al., 2001; Baloyannis, 2011). The mitochondrial import channel (TOM40) has been implicated in $\mathrm{AD}$ as an important site 
of amyloid precursor protein (APP) accumulation, which in turn is thought to trigger downstream events leading to an increase in reactive oxygen species $\left(\mathrm{H}_{2} \mathrm{O}_{2}\right)$ and mitochondrial dysfunction. Interestingly, APP accumulation within the mitochondrial import channels was found across cholinergic, dopaminergic, glutamatergic, and GABAergic neuron types in AD and was more abundant in frontal cortex and the HC (Devi et al., 2006). However, we do not know whether TOMM40 SNPs influence the channel forming subunit that is implicated as a site where APP accumulates. Nonetheless, it is possible that genetic variation in or close to TOMM40 changes the function of the TOM40 protein, thereby influencing mitochondrial functioning in areas such as the HC (Ferencz et al., 2012), but this proposed mitochondrial disconnection hypothesis has yet to be confirmed.

Recently, TOMM40 rs2075650 was associated with AD, and deemed the second most significant $\mathrm{AD}$ marker in the APOE region (Harold et al., 2009; Feulner et al., 2010). The strong Genome Wide Association Study (GWAS) signal for TOMM40 rs2075650 is interpreted as an APOE hit, due to their strong LD, and often the biological implications are considered to originate from APOE. Interestingly, the rs2075650, rs11556505 (TOMM40) and rs429358 (APOE) haplotype show greater genome wide association with AD than TOMM40 rs20756505 alone (Potkin et al., 2009). HC atrophy is common, albeit not always present, in the preclinical course of AD (Reitz et al., 2011; Tondelli et al., 2011; Lindberg et al., 2012) and the APOE $\varepsilon 4$ allele has been shown to influence HC atrophy in prodromal AD (Jack et al., 2012). TOMM40 may have an additional influence on this trajectory, as TOMM40 rs2075650 has been associated with HC integrity (Shen et al., 2010; Bis et al., 2012; Vounou et al., 2012). A recent GWAS on imaging phenotypes by Shen and colleagues (2010) found that both APOE and TOMM40 were associated with multiple imaging phenotypes. Interestingly, TOMM40 rs2075650 was specifically associated with $\mathrm{HC}$ and right amygdala volume, in comparison to the $A P O E \& 4$ allele that was linked to volumes throughout the brain including neocortical areas (parietal and temporal cortices) and the hippocampus. Thus, TOMM40 may have a more specific role within the medial-temporal lobe compared to $A P O E$, which may apply primarily to TOMM40 rs2075650 G, and possibly to rs11556505 T.

It is well-established that HC integrity is pivotal to EM performance (Milner, 1972; Tulving and Markowitsch, 1998; Squire et al., 2004). Whereas the $\varepsilon 4$ allele of the APOE gene has been linked to impaired EM performance (Small et al., 2004; Nilsson et al., 2006; De Jager et al., 2012), the influence of TOMM40 SNPs on memory has rarely been investigated. Yet, the TOMM40 poly$\mathrm{T}$ length has been found to negatively influence EM (Johnson et al., 2011), but this influence may not be independent of APOE (Schiepers et al., 2012), nor always replicable (Cruchaga et al., 2011). Recently, the TOMM40 poly-T length was found to independently modulate the adverse effects of lorazepam-induced memory impairment in aging (Pomara et al., 2011a). It remains to be determined whether TOMM40 rs11556505 and rs2075650 SNPs influence EM in normal aging.

In the past years, the impact of genes on brain and cognition has been highlighted (Rasch et al., 2010; Wilson et al., 2011), with increasing focus on the synergistic effects of different single nucleotide polymorphisms (SNPs) (Eichler et al., 2010). Thus, the primary aim of this study was to assess the influence of variations in the TOMM40 gene on HC volume and EM in old adults $(>60)$ without dementia, taking into account $A P O E$ genotype status. We analyzed two TOMM40 SNPs (rs11556505 and rs2075650), which have previously been associated with onset of AD (Grupe et al., 2007; Harold et al., 2009; Potkin et al., 2009; Feulner et al., 2010) and HC integrity (Shen et al., 2010; Bis et al., 2012; Vounou et al., 2012). We hypothesized that these TOMM40 SNPs have an influence on $\mathrm{HC}$ volume and EM performance, irrespective of APOE status, with rs2075650 G and rs11556505 T "risk alleles" negatively influencing the dependent variables.

\section{MATERIALS AND METHODS ETHICS STATEMENT}

For the current analyses a subsample of participants from the baseline assessment of the Swedish National Study on Aging and Care in Kungsholmen (SNAC-K) was used. The SNAC-K study complies with the declaration of Helsinki, and has been approved by the ethical committee at Karolinska Institutet. All subjects gave informed consent, and in the case of severe cognitive impairment consent was collected from next-of-kin.

\section{STUDY SAMPLE}

The SNAC-K study commenced in 2001, inviting randomly selected participants from the island of Kungsholmen in central Stockholm to examine medical, psychological, and social aspects of health in late adulthood (Laukka et al., 2013). Three thousand three hundred and sixty three individuals participated in baseline assessments that consisted of neuropsychological testing, medical screening and nurse interviews. The participants were randomly selected from eleven age cohorts: $60,66,72,78,81,84$, $87,90,93,96$, and 99 years and older. For the current analyses, we used a sample of 555 randomly selected non-institutionalized individuals from SNAC-K, who underwent magnetic resonance imaging (MRI). DNA and neuropsychological assessments were available for 496 of these individuals. After exclusions based on the following criteria: age 90 and above $(n=14)$, major depressive episode according to DSM-IV $(n=1)$, MMSE $\leq 24(n=$ $2)$, Guillain-Barré syndrome $(n=1)$, epilepsy $(n=2)$, alcohol dependence syndrome $(n=3)$, dyslexia $(n=3)$, stroke $(n=19)$, and suboptimal MRI image quality $(n=27)$, the final study sample comprised 424 individuals between 60 and 87 years of age. The mean age of the participants was 69.91 years $(S D=8.63)$; they had received on average 12.61 years of schooling, had a mean Mini-Mental State Examination score of 29.17 ( $S D=1.00)$, and the majority of the sample was female (59.30\%).

\section{MRI ACQUISITION AND VOLUMETRIC MEASUREMENT}

MRI data were acquired on a $1.5 \mathrm{~T}$ scanner (Philips Intera, Netherlands). 3D fast field echo (FFE) T1, axial SE (spin echo) Proton Density/T2, axial FLAIR (fluid-attenuated inversion recovery), and axial diffusion tensor imaging data were acquired. The 3D FFE T1 images $(T R=15 \mathrm{~ms}, T E=7 \mathrm{~ms}$, Flip angle $=15^{\circ}$, number of axial slices $=128$ with thickness $=$ $1.5 \mathrm{~mm}$ and in plane resolution $0.9375 \times 0.9375$, no gap, Field Of View $=240 \times 240$, matrix $=196 \times 256$ ) were used for 
volumetry. Volumetric measurements of the $\mathrm{HC}$ were performed manually on the HERMES workstation (Nuclear Diagnostics, Stockholm, Sweden) by a radiologist who was blind to the participants' genotype status. The HC formation was defined as gray matter including the HC proper (Ammon's horn), the dentate gyrus, the subiculum and some white matter (alveus, fimbria). The parahippocampal gyrus, entorhinal cortex, and fornix were excluded (Zhang et al., 2010). Intracranial volume (ICV) was acquired using STEREOLOGY (Sheline et al., 1996) in the HERMES workstation and included total brain, dura, ventricles, extraventricular CSF, brain stem, and cerebellum. Intra-rater reliability of 15 randomly selected subjects provided intraclass correlation coefficients above 0.93 for the $\mathrm{HC}$ and ICV. Left $\mathrm{HC}$ volume $\left(M=3.33 \mathrm{~cm}^{3}, S D=0.50\right)$ and right $\mathrm{HC}$ volume $\left(M=3.41 \mathrm{~cm}^{3}, S D=0.48\right)$ were highly correlated $(r=0.83$, $p<0.00)$. Thus, volumes from both hemispheres were combined, yielding an overall $\mathrm{HC}$ volume of $\left(M=6.74 \mathrm{~cm}^{3}, S D=\right.$ 0.89 ). We corrected for head size in all analyses by adding ICV as a covariate.

\section{NEUROPSYCHOLOGICAL ASSESSMENT}

EM was measured using a 16-item word list. Sixteen unrelated concrete nouns were presented to participants both visually and orally. Presentation rate was $5 \mathrm{~s}$ per word followed by a 2 -min free recall period. We used total number of words recalled as a measure of free recall.

\section{GENOTYPING}

DNA was extracted from peripheral blood samples using standard methods. Genotyping was performed using MALDI-TOF analysis on the Sequenom MassARRAY ${ }^{\mathrm{TM}}$ platform at the Mutation Analysis Facility, Karolinska Institutet. DNA was amplified using primer pairs to identify SNP loci, followed by assessing the allele-specific extension products by their respective mass, using MassARRAY MALDI-TOF mass spectrometry. Quality control was performed at the DNA-sample level, assay level, and the level of multiplex assay pool. The call rates for the SNPs included in this study were between 99.4 and $99.7 \%$. Concordance analysis was done on 486 DNA samples that were present twice, and 14 DNA samples that were present in triplets, and analyzed in separate runs. Analysis performed on all assays passing the quality controls, yielded a concordance score $>99 \%$.

We targeted two TOMM40 polymorphisms: rs11556505 and rs2075650 and for APOE we assessed the rs429358 SNP that determines $\varepsilon 4$ status. Dichotomized variables were created for APOE rs429358 (no $\varepsilon 4$ vs. any $\varepsilon 4$ ) and TOMM40 rs11556505 (CC vs. any T) and rs2075650 (AA vs. any G). Allelic frequencies
(Table 1) were in line with previous reports and Hardy Weinberg Equilibrium was $p=0.83$ for rs429358, $p=0.01$ for $\mathrm{rs} 11556505$ and $p=0.01$ for rs2075650, all considered within the range of established conventions. LD was calculated between TOMM40 and APOE SNPs using the statistical package SNPassoc in $\mathrm{R}$ (Gonzalez et al., 2007). In the current sample TOMM40 SNPs were in $\operatorname{LD}\left(D_{\max }^{\prime}=1\right)$, with $A P O E$. Furthermore, the two TOMM40 SNPs (rs11556505 and 2075650) were in high LD $\left(D^{\prime}=1\right)$. However, their frequency was not completely overlapping and, therefore, they were considered separately in the analyses.

\section{STATISTICAL ANALYSES}

We used IBM SPSS 19 for all analyses. Primary analyses to evaluate the genetic influence on EM included a series of analysis of variance (ANOVAs), with dichotomized genotype groups as factors i.e., APOE rs429358 (no $\varepsilon 4$ vs. any $\varepsilon 4$ ) and TOMM40 rs11556505 (CC vs. any T) and EM free recall as dependent variable. This way both main and interaction effects of $A P O E$ and TOMM40 were facilitated. To assess the influence of the APOE and TOMM40 SNPs on HC volume a series of analysis of covariance (ANCOVA) were conducted, with ICV as a covariate. Second order partial correlations, controlling for age and ICV, were conducted in order to assess the association between EM and $\mathrm{HC}$ volume. To ascertain if there was a statistical difference between correlation coefficients Fisher's z transformation (Fisher, 1915) was utilized.

\section{RESULTS}

\section{THE INFLUENCE OF APOE AND TOMM40 ON EM}

$2 \times 2$ ANOVAs were performed to assess the influence of $A P O E$ and TOMM40 on EM free recall, one model per TOMM40 SNP. Demographic variables are displayed in Table 2 and differences were analyzed using ANOVAs. There was no main effect of the APOE polymorphism on free recall performance (Figure 1A), meaning that presence or absence of the $\varepsilon 4$ allele had no influence on EM in the current sample $\left[F_{(1,409)}=0.49\right.$, $p=0.486]$. Furthermore, neither TOMM40 SNPs, rs20756505 $\left[F_{(1,409)}=0.29, p=0.589\right]$ nor $\operatorname{rs} 11556505\left[F_{(1,408)}=0.29\right.$, $p=0.589]$, had a significant influence on free recall performance (Figures 1B,C). Finally, we observed no interaction effects between the APOE and TOMM40 SNPs on the dependent variable free recall $(1) \operatorname{rs} 20756505\left[F_{(1,409)}=0.25, p=0.621\right],(2)$ rs11556505 $\left[F_{(1,408)}=0.23, p=0.632\right]$. The same pattern was observed that is no main or interaction effects of $A P O E$ and TOMM40 SNPs, when age, sex and education were adjusted for using ANCOVAs ( $p s>0.10)$.

Table 1 | Polymorphic characteristics of the APOE and TOMM40 gene.

\begin{tabular}{llllll}
\hline SNP & Gene & Chr & Position & Frequencies & Protein \\
\hline rs429358 & APOE & 19 & 45411941 & any $\varepsilon 427.2 \%$ & Apolipoprotein E \\
rs2075650 & TOMM40 & 19 & 45395619 & any G $27.9 \%$ & Tom40 \\
rs11556505 & TOMM40 & 19 & 45396144 & any T 27.5\% & Tom40 \\
\hline
\end{tabular}

LD, Linkage Disequilibrium measured in D'; TOMM40, Translocase of outer mitochondrial membrane 40; APOE, Apolipoprotein E; Chr, Chromosome. 
Table 2 | Demographic variables across APOE and TOMM40 status.

\begin{tabular}{|c|c|c|c|c|c|c|c|c|c|c|}
\hline TOMM40 & \multicolumn{4}{|c|}{$A P O E$ no $\varepsilon 4$} & \multicolumn{4}{|c|}{$A P O E$ any $\varepsilon 4$} & $\boldsymbol{F}$ & $P$ value \\
\hline Age & 69.80 & 8.5 & 71.73 & 9.5 & 70.69 & 9.4 & 69.88 & 8.9 & 0.52 & 0.67 \\
\hline Education & 12.57 & 4.5 & 11.28 & 3.5 & 12.50 & 4.2 & 13.27 & 4.7 & 1.53 & 0.21 \\
\hline MMSE & 29.24 & 0.9 & 28.97 & 0.9 & 29.04 & 1.2 & 29.02 & 1.1 & 1.68 & 0.17 \\
\hline rs11556505 & \multicolumn{2}{|c|}{ CC $n=272$} & \multicolumn{2}{|c|}{ Any $T n=30$} & \multicolumn{2}{|c|}{$\mathrm{CC} n=29$} & \multicolumn{2}{|c|}{ Any $T n=82$} & & \\
\hline Age & 69.87 & 8.5 & 71.73 & 9.5 & 70.69 & 8.0 & 69.83 & 9.0 & 0.49 & 0.69 \\
\hline Education & 12.57 & 4.5 & 11.28 & 3.5 & 12.41 & 4.3 & 13.31 & 4.7 & 1.61 & 0.19 \\
\hline MMSE & 29.24 & 0.9 & 28.97 & 0.9 & 29.00 & 1.2 & 29.03 & 1.1 & 1.69 & 0.17 \\
\hline Sex M/F & \multicolumn{2}{|c|}{$109 / 163$} & \multicolumn{2}{|c|}{$13 / 17$} & \multicolumn{2}{|c|}{$11 / 18$} & \multicolumn{2}{|c|}{$33 / 49$} & $0.19^{\dagger}$ & 0.98 \\
\hline
\end{tabular}

$t=\chi^{2} ;$ MMSE, Mini Mental State Examination; TOMM40, Translocase of outer mitochondrial membrane 40; APOE, Apolipoprotein E.
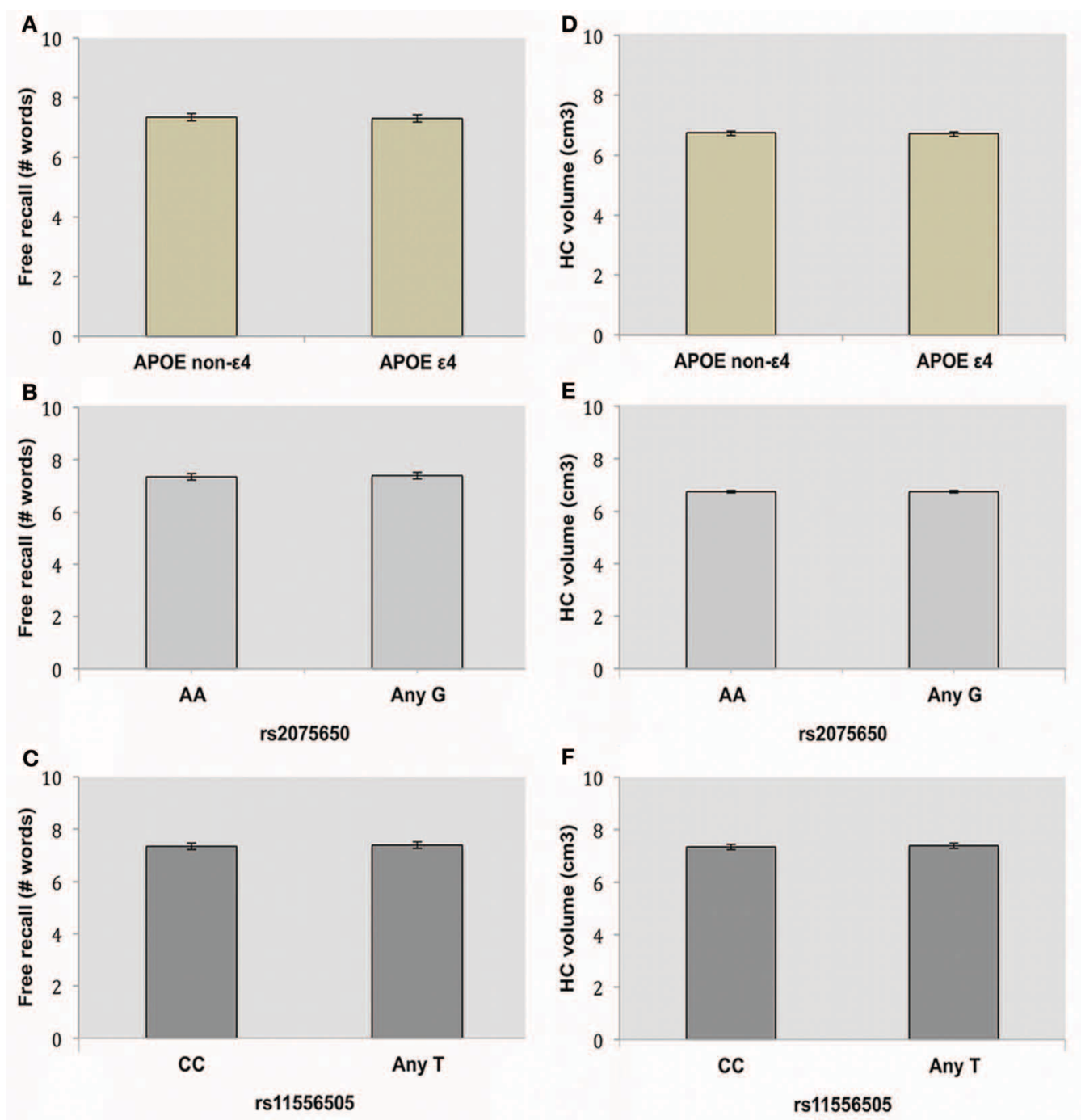

FIGURE 1 | Influence of the APOE and TOMM40 genes on episodic memory. Figures depict mean and standard deviation for free recall performance (Number of words) and hippocampal (HC) volume $\left(\mathrm{cm}^{3}\right)$ in APOE (A,D), TOMM40 rs20756505 (B,E) and rs11556505 (C,F). 


\section{THE INFLUENCE OF APOE AND TOMM40 ON HC VOLUME}

In order to assess the influence of the APOE and TOMM40 SNPs on $\mathrm{HC}$ volume we conducted $2 \times 2$ ANCOVAs, one per TOMM40 SNP, with HC volume as dependent variable. We included ICV as a covariate in the model to ensure that there were no confounding effects on HC volume as a result of variation in ICV. Running these ANCOVAs with APOE (rs429358) and TOMM40 (rs2075650 and rs11556505) resulted in no significant main effect of $A P O E\left[F_{(1,409)}=0.58, p=0.447\right]$, or TOMM40 rs20756505 $\left[F_{(1,409)}=0.07, p=0.799\right], \operatorname{rs} 11556505\left[F_{(1,408)}=0.01 p=\right.$ $0.918]$ and no interaction effects (1) $\operatorname{rs} 20756505\left[F_{(1,409)}=\right.$ $0.54 p=0.462],(2) \operatorname{rs} 11556505\left[F_{(1,408)}=0.30 p=0.583\right]$ (Figures 1D-F). This suggests that neither APOE nor TOMM40 polymorphisms have a significant influence on $\mathrm{HC}$ volume in individuals with no known neurodegenerative disorders, age 60-87. Again, no main or interaction effects were observed when controlling also for age, sex and education ( $p$ s $>0.10)$.
To test if TOMM40 genotypes influenced the association between EM and $\mathrm{HC}$ volume we conducted a series of partial correlations between free recall and HC volume. All analyses were controlled for age, which is known to influence $\mathrm{HC}$ volume in the SNAC-K sample (Zhang et al., 2010), as well as EM (Rönnlund et al., 2005), and ICV (Sanfilipo et al., 2004). The correlations were conducted in two steps; first analyzing the influence of $A P O E$ (non- $\varepsilon 4$ vs. any $\varepsilon 4$ ), followed by the influence of TOMM40 rs2075650 (AA vs. any G) and rs11556505 (CC vs. any T) in APOE \&4 carriers only. We observed a significant correlation between $\mathrm{HC}$ volume and free recall performance in $A P O E$ $\varepsilon 4$ carriers $(r=0.21, p=0.026)$, such that those carrying the $\varepsilon 4$ allele appear to require larger $\mathrm{HC}$ volume in order to perform well on free recall (Tulving and Craik, 2000), accounting for $4 \%$ of the variance (Figure 2 B). This association was not significant in $A P O E$ non- $\varepsilon 4$ carriers $(r=0.06, p=0.284)$ (Figure $2 \mathrm{~A}$ ), and when tested with the Fisher $\mathrm{z}$-transformation there was a
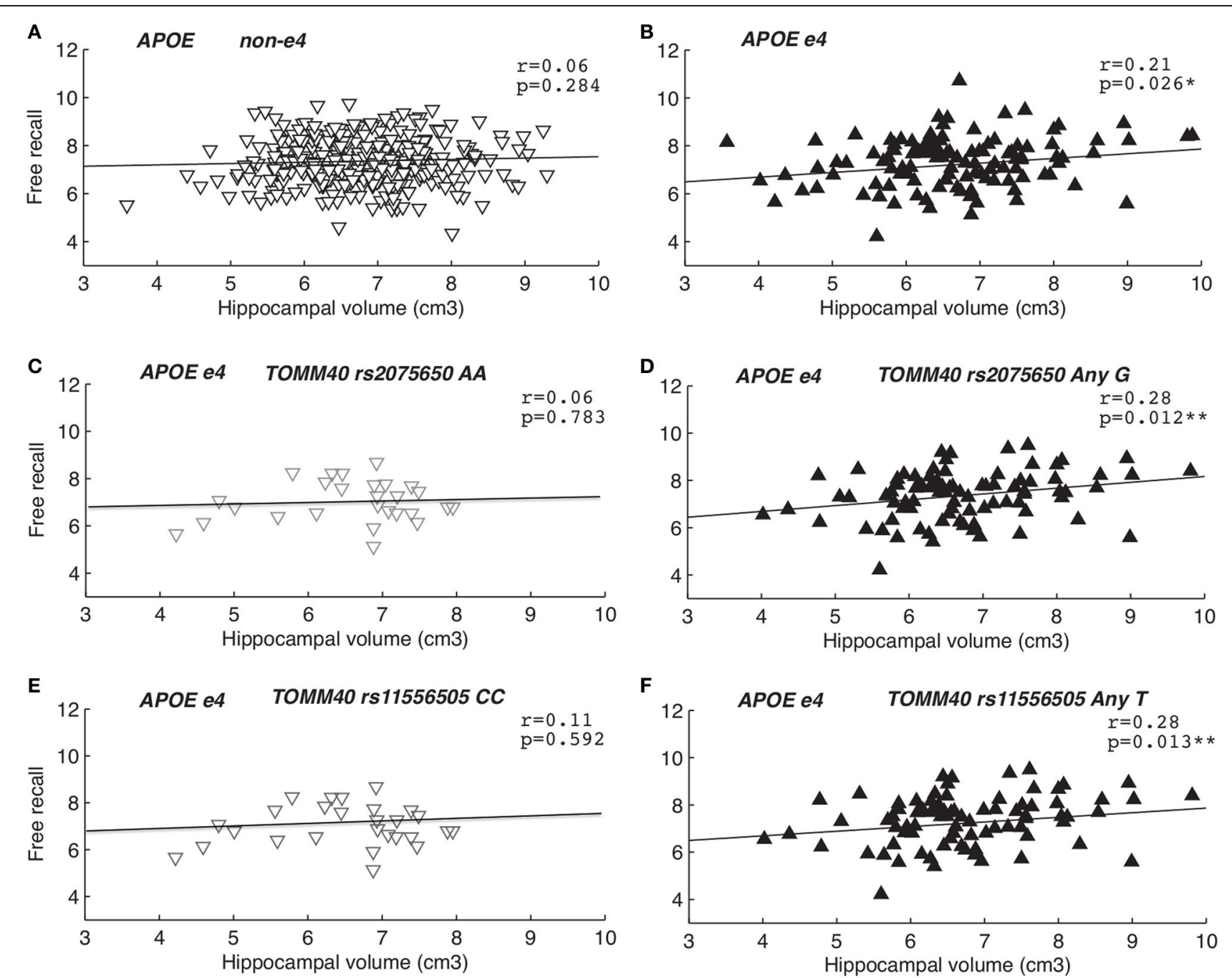

FIGURE 2 | The association between hippocampal volume and episodic memory. Second order partial correlations, between free recall performance and hippocampal volume, controlling for age and intracranial volume. Panels $(\mathbf{A}, \mathbf{B})$ depicts initial partial correlations for

$A P O E$ non- $\varepsilon 4$ vs. $\varepsilon 4$ carriers, followed by partial correlations in $A P O E \& 4$ carriers only according to TOMM40 dichotomization: (C) rs2075650 AA, (D) rs2075650 any G, (E) rs11556505 CC, (F) rs11556505 any $T$. 
non-significant trend for differences between the correlations for any $\varepsilon 4$ and non- $\varepsilon 4$ carriers $(p=0.09)$. Taking this association one step further, we assessed whether the positive correlation between $\mathrm{HC}$ volume and free recall was influenced by the presence or absence of TOMM40 "risk" alleles in APOE $\varepsilon 4$ carriers only (Figures 2D,F). The positive correlation between EM and HC volume was present among TOMM40 "risk" allele carriers rs2075650 (any G: $r=0.28, p=0.012$ ) and TOMM40 rs11556505 (any T: $r=0.28, p=0.013$ ) carriers with any APOE $\varepsilon 4$ allele, explaining $8 \%$ of the variance, in comparison to "non-risk" allele carriers rs2075650 (AA: $r=0.16, p=0.420$ ) and rs11556505 (CC: $r=0.19, p=0.338$ ) with an APOE $\varepsilon 4$ allele. Moreover, TOMM40 SNPs had no additional influence on the non-significant association among APOE non- $\varepsilon 4$ carriers ( $p s>0.10$ ). These findings were replicated when also controlling for a sex and education [APOE \&4 carriers and TOMM40 rs2075650 (any G: $r=0.28$, $p=0.012$ ) and TOMM40 rs11556505 (any T: $r=0.28, p=$ $0.012)]$. In order to correct for multiple comparisons we utilized Bonferroni correction whereby the conventional level of significance $(p<0.05)$ was divided by the number of SNPs utilized $(n=3)$, yielding a more stringent $\alpha$-level of $p<0.016$. Whereas the association between $\mathrm{HC}$ volume and free recall performance among $A P O E \& 4$ carriers did not survive correction, the association among $A P O E \& 4$ carriers with TOMM40 risk alleles remained significant (Figures 2D,F).

Upon closer examination of the correlation coefficients using Fisher z transformation (Fisher, 1915) we observed that the correlations were not significantly different in TOMM40 "risk" vs. "non-risk" allele carriers with the presence of an APOE $\varepsilon 4$ allele. Removal of one influential case ( $>2$ SD HC volume and EM memory) in APOE $\varepsilon 4$ carriers with an additional TOMM40 "nonrisk" allele (Figures 2C,E), resulted in a reduction of the magnitude of the correlation coefficient from $r=0.16$ to $r=0.06$ for rs2075650 (AA) and from $r=0.19$ to $r=0.11$ for $r s 11556505$ (CC). Note that data are presented following removal of outliers. Nevertheless, even with this reduced sample, Fishers $z$ test (Fisher, $1915)$ resulted in no significant difference in correlation coefficients across TOMM40 genotypes ( $p s>0.10$ ). No other outliers were present in the sample.

Finally, we confirmed the specificity of the association between HC volume and free recall, using a battery of tasks assessing semantic memory, verbal fluency and perceptual speed (Laukka et al., 2013). For none of these task domains, the relationship attained significance $(p s>0.10)$. All findings were replicated following exclusion of participants $(n=12)$ with a preliminary dementia diagnosis at follow-up, which took place 3 years after baseline in the old cohorts (age $\geq 78$ at baseline) and 6 years after baseline (age 60-78 at baseline), to ensure that the results were not biased by the presence of preclinical AD cases.

\section{DISCUSSION}

The aim of the current study was to assess whether there is a differential influence of APOE and TOMM40 polymorphisms on $\mathrm{HC}$ volume and EM in non-demented older adults. Our findings suggest that the APOE and TOMM40 SNPs examined do not influence $\mathrm{HC}$ volume and $\mathrm{EM}$ performance in adults above 60 years of age.
Initial analyses revealed that there was no influence of the $A P O E \& 4$ allele on free recall performance, which is in line with previous research showing limited influence of $A P O E \& 4$ on cognition in individuals free of dementia (Small et al., 2000), especially when accounting for preclinical dementia (Savitz et al., 2006; Laukka et al., 2013). Thus, our results do not provide support for a significant effect of the APOE $\varepsilon 4$ allele on $\mathrm{HC}$ volume and EM. Nonetheless, the primary aim of the current study was to gauge the influence of TOMM40 SNPs on free recall and HC volume. Although TOMM40 rs2075650 has been associated with $\mathrm{HC}$ volume in AD (Shen et al., 2010), we observed no association between either TOMM40 rs11556505 nor rs2075650 on $\mathrm{HC}$ volume in this sample of old cognitively normal individuals. Likewise, we did not find an association between TOMM40 SNPs and EM performance. Thus, it appears that these TOMM40 SNPs have no influence on HC volume and EM performance in non-demented older adults as measured in this study.

Currently, the majority of research concerning the influence of TOMM40 on cognition has been focused on the TOMM40 poly$\mathrm{T}$ repeat (rs10524523). Recently, Hayden and colleagues (2012) observed that TOMM40 length variation within the rs10524523 SNP influenced cognition in normal aging, even when accounting for APOE. In the current sample, neither TOMM40 polymorphisms (rs2075650, rs11556505) had a direct influence on cognition. Yet it is possible that the differential effect of $A P O E$ and TOMM40 is age-dependent and therefore not present in the current sample. A recent longitudinal study by Caselli and colleagues (2012) demonstrated that variation in the TOMM40 poly-T length influences memory performance independently of $A P O E$ variation prior to the age of 60 . Moreover, our understanding of the biological expression of TOMM40 SNPs remains limited. Indeed, a recent study by Hedskog and colleagues (2012) found that the TOMM40 rs10524523 repeat length polymorphism (poly-T), had no detectable influence on mitochondrial morphology when measured in fibroblasts in healthy individuals before 64 years of age. However, there is a lack of knowledge regarding the influence of other TOMM40 polymorphisms on mitochondrial functioning and morphology in individuals above this age. A recent study by Bekris and colleagues (2012) showed that regions within TOMM40, including TOMM40 poly-T and rs2075650, are involved in APOE and TOMM40 gene regulation by influencing promoter activity in certain haplotype structures depending on haplotype and cell type. These findings suggest that gene expression within the TOMM40 region is complex and haplotype- as well as cell-dependent. However, despite some implications from the literature of a TOMM40 component to EM and $\mathrm{HC}$ volume, we were not able to confirm this association with the current TOMM40 SNPs in our population based sample of non-demented older individuals.

Whereas, a direct influence of TOMM40 variation was not evident in the current study, we observed a significant association between $\mathrm{HC}$ volume and free recall performance in APOE \&4 carriers who also carried TOMM40 "risk alleles." The relationship between $\mathrm{HC}$ volume and $\mathrm{EM}$ in $A P O E \& 4$ carriers with the two TOMM40 "risk alleles" were the only correlation that survived correction for multiple testing. Moreover, the association between $\mathrm{HC}$ volume and free recall in APOE $\& 4$ carriers with TOMM40 
"non-risk alleles" was markedly reduced upon the removal of an outlier, strengthening our hypothesis that the strongest association is found in APOE \&4 carriers with TOMM40 "risk alleles." Although the association was primarily present in APOE $\varepsilon 4$ carriers who also carry TOMM40 "risk alleles" it should be noted that the correlation coefficients in question were not significantly different and the lack of association in APOE $\varepsilon 4$ carriers with TOMM40 "non-risk alleles" may reflect small sample size in this group.

Nevertheless, the positive association between $\mathrm{HC}$ volume and free recall performance indicates that $A P O E \& 4$ allele carriers who also carry a TOMM40 "risk allele" may be more dependent on $\mathrm{HC}$ volume for proficient EM performance, in comparison to "non-risk" allele carriers of these polymorphisms. Although both TOMM40 SNPs were driving the association between HC volume and EM memory performance in $A P O E \& 4$ carriers, the two TOMM40 SNPs are in perfect LD. However, the frequencies of TOMM40 SNPs are not completely overlapping.

Our findings indicate that, although variation within the TOMM40 gene does not directly influence HC volume, the polymorphisms seem to have an effect on $\mathrm{HC}$ functioning, beyond that of $A P O E \varepsilon 4$ alone. This finding lends support to the mitochondrial disconnection hypothesis (Ferencz et al., 2012), suggesting that there may be a mitochondrial component of $\mathrm{HC}$ functioning in normal aging, with a possible TOMM40-induced oxidative stress in $\mathrm{HC}$ neurons that does not influence volume per $s e$, but rather the function of this brain structure in old adults. This result is in line with data demonstrating an independent influence of TOMM40 polymorphisms on markers of neuronal damage. Higher levels of neurofilament light proteins, a sign of neuronal damage, have been found in the cerebrospinal fluid of cognitively intact individuals, but only among APOE $\varepsilon 4$ carriers who do not carry the short poly-T variant of TOMM40, a variant that is considered protective (Bruno et al., 2012). APOE $\varepsilon 4$ alone did not influence markers of neuronal damage suggesting that it is the negative combination of the two genes that is associated with neuronal damage. Although we did not test markers of neuronal damage in our current sample, our findings indicate that $A P O E \& 4$ may be potentially detrimental to HC functioning only in combination with TOMM40 "risk alleles." This hypothesis will have to be confirmed in future fMRI studies.

Other studies focusing on the TOMM40 poly-T length have shown that TOMM40 poly-T length has limited influence on mitochondrial morphology (Hedskog et al., 2012), and levels of amyloid and tau in cerebrospinal fluid (Pomara et al., 2011b), suggesting that TOMM40-modulated neurotoxicity may not be markedly present in healthy aging. Thus, in non-pathological aging, where HC volume and EM are relatively intact (Kalpouzos et al., 2009; Nyberg et al., 2012), mitochondrial morphology may be well-preserved among TOMM40 "risk" allele carriers, in line with the lack of direct influence of TOMM40 SNPs on medial temporal lobe integrity observed in the current sample. That said, there may already be signs of TOMM40-induced neuronal damage in old adults (Bruno et al., 2012), accounting for the added influence of TOMM40 (rs11556505 any T allele, rs2075650 any $\mathrm{G}$ allele) on the association between HC integrity and free recall in $A P O E \& 4$ carriers observed here. Future studies in healthy populations taking in consideration markers of neuronal damage and oxidative stress should confirm this hypothesis.

One could argue that the association we observed is primarily dependent on $A P O E \& 4$, as APOE and the current TOMM40 SNPs were in LD. However, the presence of an association among APOE $\varepsilon 4$ carriers with TOMM40 "risk alleles" speaks against this notion. Thus, despite high LD between TOMM40 rs20756505 and rs1155650 and APOE, there appears to be an added influence of TOMM40 "risk alleles" on the HC volume-EM link.

\section{LIMITATIONS}

Our findings should be considered in light of some limitations. Although we have a large sample, power concerns may still apply with regard to the effects of the specific genes on brain and cognition. Post-hoc power calculations were conducted utilizing G*Power3 (Faul et al., 2007). Based on the assumption that the effect size for the genetic influence on memory performance and HC volume is $\eta^{2}=0.1$ (Rasch et al., 2010; Laukka et al., 2013), our sample of $n=424$ individuals provides sufficient, albeit low, power $(60 \%)$ to detect a significant difference $(\alpha=0.05)$ in memory performance between genotype groups. In addition, our sample size was too small to examine potential dose-response effect for the APOE $\varepsilon 4$ allele. We did not observe any direct influence of APOE and TOMM40 polymorphisms in the current sample. Yet, it has been suggested that, in order to tease out the true effects in this region that is in $\mathrm{LD}$, sample sizes of up to 20000 would be required (Guerreiro and Hardy, 2012).

There is an inherent limitation in the cross-sectional nature of our study and future studies should assess if TOMM40 SNPs are associated with longitudinal changes. The current sample was too small to assess the gene-memory link per age-cohort but future studies with larger sample sizes should address this matter. Thus, future longitudinal studies with larger sample sizes should consider the independent influence of APOE and TOMM40 on rate of brain and memory change, and how this relationship is expressed in different age cohorts. Potential confounders that may have influenced our findings include age, sex, and education. However, our findings were replicated after controlling for these confounding factors. Moreover, we limited the potential influence of incipient dementia by replicating our findings following exclusion of preclinical dementia cases, suggesting that incipient dementia had no influence on the current findings.

\section{CONCLUDING REMARKS}

In conclusion, we found no influence of APOE and TOMM40 polymorphisms on $\mathrm{HC}$ volume and EM in old adults suggesting that variation in these genes has limited influence on brain and cognition in non-demented adults above age 60. Our findings, however, provide some, albeit limited, support for an added influence of TOMM40 polymorphisms (rs2075650, rs11556505) on EM in normal aging. The aforementioned findings of the current study suggest that individuals with the APOE $\varepsilon 4$ allele in combination with TOMM40 rs2075650 and rs11556505 "risk-alleles" may have greater reliance on $\mathrm{HC}$ volume for adequate EM performance. Whether the influence of the TOMM40 gene is more 
pivotal in prodromal $\mathrm{AD}$ remains to be seen and future followup examination of the study sample should provide evidence that speaks to this issue.

\section{ACKNOWLEDGMENTS}

We are grateful to all the participants, caregivers and staff involved in the SNAC-K study. This research has made use of

\section{REFERENCES}

Abraham, R., Moskvina, V., Sims, R., Hollingworth, P., Morgan, A., Georgieva, L., et al. (2008). A genome-wide association study for late-onset Alzheimer's disease using DNA pooling. BMC Med. Genomics 1:44. doi: 10.1186/1755-8794-1-44

Baloyannis, S. J. (2011). Mitochondria are related to synaptic pathology in Alzheimer's disease. Int. J. Alzheimers Dis. 2011:305395. doi: 10.4061/2011/305395

Bekris, L. M., Lutz, F., and Yu, C. E. (2012). Functional analysis of APOE locus genetic variation implicates regional enhancers in the regulation of both TOMM40 and APOE. J. Hum. Genet. 57, 18-25.

Bis, J. C., DeCarli, C., Smith, A. V., van der Lijn, F., Crivello, F., Bis Fornage, M., et al. (2012). Common variants at $12 \mathrm{q} 14$ and $12 \mathrm{q} 24$ are associated with hippocampal volume. Nat. Genet. 44, 545-551.

Bruno, D., Pomara, N., Nierenberg, J., Ritchie, J. C., Lutz, M. W., Zetterberg, H., et al. (2012). Levels of cerebrospinal fluid neurofilament light protein in healthy elderly vary as a function of TOMM40 variants. Exp. Gerontol. 47, 347-352.

Caselli, R. J., Dueck, A. C., Huentelman, M. J., Lutz, M. W., Saunders, A. M., and Reiman, E. M. (2012). Longitudinal modeling of cognitive aging and the TOMM40 effect. Alzheimers Dement 8, 490-495.

Cruchaga, C., Nowotny, P., Kauwe, J. S., Ridge, P. G., Mayo, K., Bertelsen, S., et al. (2011). Association and expression analyses with singlenucleotide polymorphisms in TOMM40 in Alzheimer disease. Arch. Neurol. 68, 1013-1019.

De Jager, P. L., Shulman, J. M., Chibnik, L. B., Keenan, B. T., Raj, T., Wilson, R. S., et al. (2012). A genome-wide scan for common variants affecting the rate of age-related cognitive decline. Neurobiol. Aging 33, e1-e15.

Devi, L., Prabhu, B. M., Galati, D. F., Avadhani, N. G., and Anandatheerthavarada, H. K. (2006). Accumulation of amyloid precursor protein in the mitochondrial import channels of human Alzheimer's disease brain is associated with mitochondrial dysfunction. J. Neurosci. 26, 9057-9068.

Eichler, E. E., Flint, J., Gibson, G., Kong, A., Leal, S. M., Moore, J. H., et al. (2010). Missing heritability and strategies for finding the underlying causes of complex disease. Nat. Rev. Genet. 11, 446-450.

Faul, F., Erdfelder, E., Lang, A. G., and Buchner, A. (2007). G* Power 3: a flexible statistical power analysis program for the social, behavioral, and biomedical sciences. Behav. Res. Methods 39, 175-191.

Ferencz, B., Karlsson, S., and Kalpouzos, G. (2012). Promising genetic biomarkers of preclinical Alzheimer's disease: the influence of APOE and TOMM40 on brain integrity. Int. J. Alzheimers Dis. 2012:421452. doi: 10.1155/ 2012/421452

Feulner, T. M., Laws, S. M., Friedrich, P., Wagenpfeil, S., Wurst, S. H., Riehle, C., et al. (2010). Examination of the current top candidate genes for $\mathrm{AD}$ in a genome-wide association study. Mol. Psychiatry 15, 756-766.

Fisher, R. A. (1915). Frequency distribution of the values of the correlation coefficient in samples of an indefinitely large population. Biometrika 10, 507-521.

Gonzalez, J. R., Armengol, L., Sole, X., Guino, E., Mercader, J. M., Estivill, X., et al. (2007). SNPassoc: an $\mathrm{R}$ package to perform whole genome association studies. Bioinformatics 23, 644-645.

Grupe, A., Abraham, R., Li, Y., Rowland, C., Hollingworth, P., Morgan, A., et al. (2007). Evidence for novel susceptibility genes for late-onset Alzheimer's disease from a genome-wide association study of putative functional variants. Hum. Mol. Genet. 16, 865-873.

Guerreiro, R. J., and Hardy, J. (2012). TOMM40 Association with Alzheimer disease: tales of $A P O E$ and linkage disequilibrium. Arch. Neurol. 69, 1243-1244.

the SMILE medical imaging laboratory at Karolinska University Hospital, Stockholm, Sweden. The current study was supported by Stiftelsen för Gamla Tjänarinnor and Sigurd och Elsa Goljes Minne, the Swedish Research Council, the Swedish Council for Working Life and Social Research, Swedish Brain Power, an Alexander von Humboldt Research Award, and a donation from the af Jochnick Foundation.

Harold, D., Abraham, R. Hollingworth, P., Sims, R. Gerrish, A., and Hamshere, M. L. (2009). Genome-wide association study identifies variants at $C L U$ and PICALM associated with Alzheimer's disease. Nat. Genet. 41, 1088-1093.

Hayden, K. M., McEvoy, J. M. Linnertz, C., Attix, D., Kuchibhatla, M., Saunders, A. M., et al. (2012). A homopolymer polymorphism in the TOMM40 gene contributes to cognitive performance in aging. Alzheimers Dement 8, 381-388.

Hedskog, L., Brohede, J., Wiehager, B. Pinho, C. M., Revathikumar, P., Lilius, L., et al. (2012). Biochemical studies of poly$\mathrm{T}$ variants in the Alzheimer's disease associated TOMM40 gene. J. Alzheimers Dis. 31 , 527-536.

Hirai, K., Aliev, G., Nunomura, A., Fujioka, H., Russell, R. L., Atwood, C. S., et al. (2001). Mitochondrial abnormalities in Alzheimer's disease. J. Neurosci. 21, 3017-3023.

Humphries, A. D., Streimann, I. C., Stojanovski, D., Johnston, A. J., Yano, M., and Hoogenraad, N. J. (2005). Dissection of the mitochondrial import and assembly pathway for human Tom40. J. Biol. Chem. 280, 11535-11543.

Jack, C. R. Jr., Vemuri, P., Wiste, H. J., Weigand, S. D., Lesnick, T. G., Lowe, V., et al. (2012). Shapes of the trajectories of 5 major biomarkers of Alzheimer disease. Arch. Neurol. 69, 856-867.

Johnson, S. C., La Rue, A., Hermann, B. P., Xu, G., Koscik, R. L., and Jonaitis, E. M. (2011). The effect of TOMM40 poly-T length on gray matter volume and cognition in middle-aged persons with APOEvarepsilon3/varepsilon3 genotype. Alzheimers Dement 7 , 456-465.

Jun, G., Vardarajan, B. N., Buros, J., Yu, C. E., Hawk, M. V., and Dombroski, B. A. (2012). Comprehensive search for Alzheimer disease susceptibility loci in the $A P O E$ region. Arch. Neurol. 69, 1270-1279.

Kalpouzos, G., Chetelat, G., Baron, J. C., Landeau, B., Mevel, K.,
Godeau, C., et al. (2009). Voxelbased mapping of brain gray matter volume and glucose metabolism profiles in normal aging. Neurobiol. Aging 30, 112-124.

Laukka, E. J., Lövdén, M., Herlitz, A., Karlsson, S., Ferencz, B., Pantzar, A., et al. (2013). Genetic effects on old-age cognitive functioning: a population-based study. Psychol. Aging 28, 262-274.

Li, G., Bekris, L. M., Leong, L. Steinbart, E. J., Shofer, J. B., Crane, P. K., et al. (2012). TOMM40 intron 6 poly-T length, age at onset, and neuropathology of $\mathrm{AD}$ in individuals with $A P O E$ varepsilon3/varepsilon3. Alzheimers Dement. doi: 10.1016/j.jalz.2012. 06.009. [Epub ahead of print].

Lin, M. T., and Beal, M. F. (2006). Mitochondrial dysfunction and oxidative stress in neurodegenerative diseases. Nature 443, 787-795.

Lindberg, O., Walterfang, M., Looi, J. C., Malykhin, N., Ostberg, P., and Zandbelt, B. (2012). Hippocampal shape analysis in Alzheimer's disease and frontotemporal lobar degeneration subtypes. J. Alzheimers Dis. 30, 355-365.

McBride, H. M., Neuspiel, M., and Wasiak, S. (2006). Mitochondria: more than just a powerhouse. Curr. Biol. 16, R551-R560.

Milner, B. (1972). Disorders of learning and memory after temporal lobe lesions in man. Clin. Neurosurg. 19, 421-446.

Nilsson, L. G., Adolfsson, R., Bäckman, L., Cruts, M., Nyberg, L., Small, B. J., et al. (2006). The influence of $A P O E$ status on episodic and semantic memory: data from a populationbased study. Neuropsychology 20 645-657.

Nyberg, L., Lövdén, M., Riklund, K., Lindenberger, U., and Bäckman, L. (2012). Memory aging and brain maintenance. Trends Cogn. Sci. 16, 292-305.

Pomara, N., Bruno, D., Sidtis, J. J., Lutz, M. W., Greenblatt, D. J., Saunders, A. M., et al. (2011a). Translocase of outer mitochondrial membrane 40 homolog (TOMM40) poly-T length modulates lorazepam-related 
cognitive toxicity in healthy APOE epsilon4-negative elderly. J. Clin. Psychopharmacol. 31, 544-546.

Pomara, N., Bruno, D., Nierenberg, J. J., Sidtis, J. J., Martiniuk, F. T., and Mehta, P. D. (2011b). TOMM40 poly-T variants and cerebrospinal fluid amyloid beta levels in the elderly. Neurochem. Res. 36, 1124-1128.

Potkin, S. G., Guffanti, G., Lakatos, A., Turner, J. A., Kruggel, F., Fallon, J. H., et al. (2009). Hippocampal atrophy as a quantitative trait in a genome-wide association study identifying novel susceptibility genes for Alzheimer's disease. PLOS ONE 4:e6501. doi: 10.1371/ journal.pone.0006501

Rasch, B., Papassotiropoulos, A., and de Quervain, D. F. (2010). Imaging genetics of cognitive functions: focus on episodic memory. Neuroimage 53, 870-877.

Reddy, P. H., Manczak, M., Mao, P., Calkins, M. J., Reddy, A. P., and Shirendeb, U. (2010). Amyloidbeta and mitochondria in aging and Alzheimer's disease: implications for synaptic damage and cognitive decline. J. Alzheimers Dis. 20, S499-S512.

Reitz, C., Brayne, C., and Mayeux, R. (2011). Epidemiology of Alzheimer disease. Nat. Rev. Neurol. 7, 137-152.

Rönnlund, M., Nyberg, L., Bäckman, L., and Nilsson, L. G. (2005). Stability, growth, and decline in adult life span development of declarative memory: cross-sectional and longitudinal data from a population-based study. Psychol. Aging 20, 3-18.

Roses, A. D., Lutz, M. W., AmrineMadsen, H., Saunders, A. M., Crenshaw, D. G., and Sundseth, S. S (2010). A TOMM40 variable-length polymorphism predicts the age of late-onset Alzheimer's disease. Pharmacogenomics J. 10, 375-384.

Savitz, J., Solms, M., and Ramesar, R. (2006). Apolipoprotein E variants and cognition in healthy individuals: a critical opinion. Brain Res. Rev. 51, 125-135.

Sanfilipo, M. P., Benedict, R. H., Zivadinov, R., and Bakshi, R. (2004). Correction for intracranial volume in analysis of whole brain atrophy in multiple sclerosis: the proportion vs. residual method. Neuroimage 22, 1732-1743.

Schiepers, O. J., Harris, S. E., Gow, A. J., Pattie, A., Brett, C. E., Starr J. M., et al. (2012). APOE E4 status predicts age-related cognitive decline in the ninth decade: longitudinal follow-up of the Lothian Birth Cohort 1921. Mol. Psychiatry 17, 315-324.

Sheline, Y. I., Black, K. J., Lin, D. Y., Christensen, G. E., Gado, M. H., Brunsden, B. S., et al. (1996). Stereological MRI volumetry of the frontal lobe. Psychiatr Res. 67 203-214.

Shen, L., Kim, S., Risacher, S. L., Nho, K., Swaminathan, S., West, J. D., et al. (2010). Whole genome association study of brain-wide imaging phenotypes for identifying quantitative trait loci in MCI and AD: a study of the ADNI cohort. Neuroimage 53, 1051-1063.
Small, B. J., Graves, A. B., McEvoy, C. L., Crawford, F. C., Mullan, M., and Mortimer, J. A. (2000). Is APOEepsilon4 a risk factor for cognitive impairment in normal aging? Neurology 54, 2082-2088.

Small, B. J., Rosnick, C. B., Fratiglioni, L., and Bäckman, L. (2004). Apolipoprotein E and cognitive performance: a meta-analysis. Psychol. Aging 19, 592-600.

Squire, L. R., Stark, C. E., and Clark, R. E. (2004). The medial temporal lobe. Annu. Rev. Neurosci. 27, 279-306.

Swerdlow, R. H. (2011). Brain aging Alzheimer's disease, and mitochondria. Biochim. Biophys. Acta 1812, 1630-1639.

Tondelli, M., Wilcock, G. K., Nichelli, P., De Jager, C. A., Jenkinson, M., and Zamboni, G. (2011). Structural MRI changes detectable up to ten years before clinical Alzheimer's disease. Neurobiol. Aging 33, 825.e25-825.e36.

Tulving, E., and Craik, F. I. M. (2000). The Oxford Handbook of Memory. Oxford, New York: Oxford University Press.

Tulving, E., and Markowitsch, H. J. (1998). Episodic and declarative memory: role of the hippocampus. Hippocampus 8, 198-204.

Vounou, M., Janousova, E., Wolz, R., Stein, J. L., Thompson, P. M. Rueckert, D., et al. (2012). Sparse reduced-rank regression detects genetic associations with voxelwise longitudinal phenotypes in Alzheimer's disease. Neuroimage 60 700-716.

Wilson, R. S., Barral, S., Lee, J. H., Leurgans, S. E., Foroud, T.
M., Sweet, R. A., et al. (2011). Heritability of different forms of memory in the Late Onset Alzheimer's Disease Family Study. J. Alzheimers Dis. 23, 249-255.

Zhang, Y., Qiu, C., Lindberg, O., Bronge, L., Aspelin, P., Bäckman, L., et al. (2010). Acceleration of hippocampal atrophy in a nondemented elderly population: the SNAC-K study. Int. Psychogeriatr. 22, 14-25.

Conflict of Interest Statement: The authors declare that the research was conducted in the absence of any commercial or financial relationships that could be construed as a potential conflict of interest.

Received: 08 March 2013; accepted: 29 April 2013; published online: 22 May 2013.

Citation: Ferencz B, Laukka EJ, Lövdén $M$, Kalpouzos G, Keller L, Graff $C$, Wahlund $L-O$, Fratiglioni $L$ and Bäckman L (2013) The influence of APOE and TOMM40 polymorphisms on hippocampal volume and episodic memory in old age. Front. Hum. Neurosi. 7:198. doi: 10.3389/fnhum.2013.00198 Copyright (c) 2013 Ferencz, Laukka, Lövdén, Kalpouzos, Keller, Graff, Wahlund, Fratiglioni and Bäckman. This is an open-access article distributed under the terms of the Creative Commons Attribution License, which permits use, distribution and reproduction in other forums, provided the original authors and source are credited and subject to any copyright notices concerning any third-party graphics etc. 